J. Phys. IV France 138 (2006) 35-41

(C) EDP Sciences, Les Ulis

DOI: 10.1051/jp4:2006138005

\title{
Étude expérimentale de l'optimisation de la génération d'harmoniques d'ordre élevé par l'utilisation d'un algorithme génétique
}

\author{
C. Valentin ${ }^{1}$, O. Boyko ${ }^{1}$, G. Rey ${ }^{1}$, B. Mercier ${ }^{1}$, E. Papalazarou ${ }^{1}$, L. Antonucci ${ }^{1}$ \\ et $\mathrm{Ph}$. Balcou ${ }^{1}$ \\ ${ }^{1}$ Laboratoire d'Optique Appliquée, CNRS, École Polytechnique - ENSTA, UMR 7639, \\ Chemin de la Hunière, 91761 Palaiseau Cedex, France
}

\begin{abstract}
Résumé. Dans le but de développer une source intense EUV pour les applications, nous avons réalisé des expériences d'optimisation du signal harmonique. La génération d'harmoniques d'ordre élevé est très sensible à la forme de l'impulsion du laser fondamental. Nous avons utilisé un filtre acoustooptique dispersif programmable (Dazzler) afin de modifier la phase spectrale du laser fondamental. Nous avons optimisé le signal harmonique généré dans l'argon à l'aide d'un algorithme génétique jusqu'à un facteur 10. Nous montrons l'influence des paramètres macroscopiques de génération sur le processus d'optimisation.
\end{abstract}

\section{INTRODUCTION}

Les harmoniques élevées générées par interaction d'un laser infrarouge avec un gaz rare constituent une source de lumière cohérente dans le domaine Extrême Ultra-Violet. Dans le but d'utiliser cette source pour les applications, nous cherchons à optimiser le signal des harmoniques. Les algorithmes génétiques ont déjà été utilisés soit pour modifier la forme temporelle des impulsions du laser fondamental afin d'optimiser le signal des harmoniques élevées [1,2] soit pour faire varier la longueur d'onde d'émission d'une harmonique [3]. Pour la première fois nous présentons une étude systématique de l'optimisation des signaux harmoniques utilisant un filtre acousto-optique dispersif programmable (Dazzler).

Dans une première partie, nous décrivons l'algorithme génétique utilisé, ainsi que les paramètres pour assurer la convergence de l'algorithme. Ensuite, nous présentons les résultats expérimentaux obtenus. Nous analysons les résultats dans un troisième paragraphe avant de conclure et de donner les perspectives de ce travail.

\section{PARAMÈTRES DE L'ALGORITHME GÉNÉTIQUE}

\subsection{Description de l'algorithme}

L'algorithme génétique est un algorithme très efficace de recherche de paramètres d'optimisation pour un problème complexe. Il est basé sur une analogie avec la théorie de l'évolution dans la nature : nous définissons des individus contenant un ensemble de gènes. A chaque génération, les individus (ensemble de gènes) peuvent se croiser et produire de nouveaux individus. Une fonction de coût permet alors de ne garder que les individus qui optimisent le signal harmonique. Nous pouvons en plus introduire un taux de mutation $\left(\tau_{\text {mut }}\right.$ ) pour ces ensembles de gènes afin d'explorer toutes les solutions possibles. Ainsi, l'algorithme converge vers la solution la plus optimisée et non vers un optimum local. 


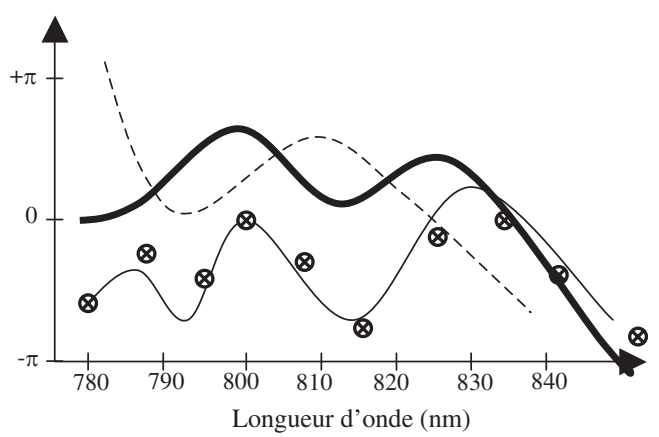

Figure 1. Phase spectrale (trait épais) calculée à partir de l'interpolation (trait fin) sur les 10 valeurs arbitraires (croix entourées) et termes d'ordre 2 et 3 du développement de Taylor (tirets).

Afin d'optimiser ce signal, nous agissons sur la phase spectrale du laser. Pour les paramètres d'optimisation, nous avons défini un individu comprenant 12 gènes. Tout d'abord, nous considérons les ordres 2 et 3 du développement de Taylor en pulsation de cette phase spectrale:

$$
\varphi(\omega)=\varphi_{0}+\varphi_{1}\left(\omega-\omega_{0}\right)+\varphi_{2}\left(\omega-\omega_{0}\right)^{2}+\varphi_{3}\left(\omega-\omega_{0}\right)^{3}+O\left(\left(\omega-\omega_{0}\right)^{3}\right)
$$

où $\varphi_{0}$ est la phase constante, $\varphi_{1}$ est le terme de délai, $\varphi_{2}$ et $\varphi_{3}$ correspondent aux termes de la dispersion de la vitesse de groupe. Nous ajoutons à ces deux paramètres 10 valeurs arbitraires définissant cette phase sur tout le spectre (cf figure 1). Pour chaque individu, l'algorithme calcule l'interpolation de cette phase. Cette phase est ensuite ajoutée à la phase définie par la série de Taylor avec une constante additive. L'onde acoustique envoyée dans le cristal biréfringent du Dazzler suit cette phase : l'impulsion laser correspondante est donc modifiée pour chaque individu.

Le spectre des harmoniques est ensuite enregistré et l'algorithme calcule la fonction de coût définie par:

$$
\mathrm{C}=\mathrm{P}_{\text {largeur }} \mathrm{C}_{\text {largeur }}+\mathrm{P}_{\text {amplitude }} \mathrm{C}_{\text {amplitude }}+\mathrm{P}_{\text {position }} \mathrm{C}_{\text {position }}
$$

Cette définition très générale de la fonction de coût nous permet d'optimiser avec le même algorithme, différentes caractéristiques du rayonnement harmonique : la largeur spectrale d'une harmonique donnée $\left(\mathrm{C}_{\text {largeur }}\right)$, l'amplitude spectrale d'une harmonique donnée $\left(\mathrm{C}_{\text {amplitude }}\right)$, ou sa longueur d'onde $\left(\mathrm{C}_{\text {position }}\right)$. Il suffit pour cela de donner un poids relatif différent à chaque terme de la fonction de coût (noté $\mathrm{P}$ ). Pour notre expérience, nous avons optimisé le signal pour une harmonique donnée. Donc, les poids relatifs pour la largeur spectrale et pour la longueur d'onde sont restés nuls dans toute notre étude.

\subsection{Choix des paramètres de l'algorithme}

Pour chaque optimisation, nous nous intéressons à seulement trois pics harmoniques du spectre (cf spectre de la figure 3). Nous définissons alors la position de chaque pic et l'amplitude à atteindre. Nous avons fait varier le poids relatif de chaque harmonique : soit les trois harmoniques ont un poids de 1 et nous optimisons le signal pour ces trois harmoniques, soit nous choisissons l'harmonique centrale avec un poids de 1 et les autres ne sont pas considérés (poids nul). Dans ce dernier cas, nous avons vu que le signal des harmoniques adjacentes évolue de manière anti-corrélée au cours de l'optimisation (cf Bartels et al. [2]). Le rapport d'intensités entre le signal de l'harmonique centrale et celui des harmoniques adjacentes est d'environ 2 à la fin de l'optimisation (cf figure 2). Nous n'avons pas observé de disparition des pics adjacents.

Nous avons aussi fait varier le taux de mutation génétique : pour un taux de $20 \%$, l'algorithme converge vers la même optimisation plus rapidement que pour un taux de $10 \%$. 


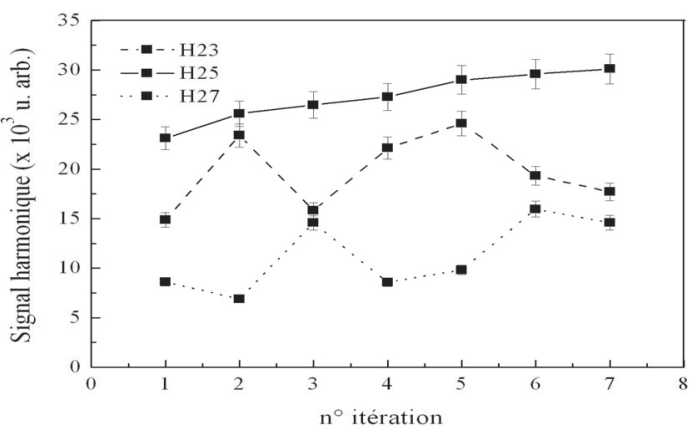

Figure 2. Optimisation du signal de l'harmonique 25 seule.

\section{RÉSULTATS EXPÉRIMENTAUX}

Dans cette partie, nous allons décrire le montage expérimental et les résultats obtenus pour chaque expérience d'optimisation.

\subsection{Description du dispositif expérimental}

Nous disposons d'un laser Ti:Sa émettant des impulsions centrée à $810 \mathrm{~nm}$, de 42 fs de durée, avec une énergie pouvant atteindre $6 \mathrm{~mJ}$ à une cadence de $1 \mathrm{kHz}$. Le col du faisceau a été mesuré et est égal à $18 \mathrm{~mm}$. La durée des impulsions a été mesurée par un SPIDER (Spectral Phase Interferometer for Direct Electric-field Reconstruction) [4] et est égale à 42 fs. Ce laser est basé sur la technique CPA (Chirped Pulse Amplification). Entre l'étireur et le premier étage d'amplification, nous avons placé un filtre acousto-optique dispersif programmable (Dazzler [5]). Ce dispositif permet de modifier l'amplitude et la phase spectrale des impulsions laser. L'impulsion laser incidente polarisée linéairement suivant l'axe ordinaire est diffractée par un réseau de Bragg crée par l'onde acoustique selon l'axe extraordinaire. Nous imposons alors le profil temporel de l'impulsion via cette onde acoustique. Le façonnage en amplitude est obtenu directement par l'efficacité de diffraction, qui dépend de la puissance spectrale acoustique contrôlable. La forme spectrale acoustique est transférée à l'impulsion optique. Pour notre expérience, nous avons modifié seulement la phase spectrale. Nous avons vérifié par une mesure SPIDER que les différents éléments de la châ̂ne laser ne modifient pas la phase spectrale imposée (chaîne linéaire).

Le faisceau ainsi caractérisé est tout d'abord diaphragmé puis focalisé à l'aide d'une lentille en $\mathrm{MgF}_{2}$, de focale $1 \mathrm{~m}$ dans une cellule remplie d'un gaz rare (Ar). L'énergie laser juste avant le diaphragme est d'environ $5 \mathrm{~mJ}$ pour toutes les expériences. Les harmoniques d'ordre élevé sont générées dans la cellule de gaz et se propagent dans la même direction que le faisceau laser IR. Afin de n'observer que les harmoniques, l'infrarouge est très fortement atténué par deux filtres en aluminium de $300 \mathrm{~nm}$ d'épaisseur chacun. Le faisceau harmonique est ensuite analysé à l'aide d'un spectromètre XUV composé d'un miroir torique en or, de focale $1 \mathrm{~m}$ et d'un réseau en incidence rasante $(600 \mathrm{tr} / \mathrm{mm})$. Nous enregistrons le signal harmonique mesuré à l'aide d'une caméra CCD XUV (cf figure 3). Ce signal est ensuite analysé par le programme d'optimisation basé sur l'algorithme génétique décrit précédemment.

\subsection{Résultats des optimisations}

Dans un premier temps, nous avons optimisé le signal sans l'algorithme génétique en faisant varier les différents paramètres de génération des harmoniques : diamètre du diaphragme, position de la lentille, 


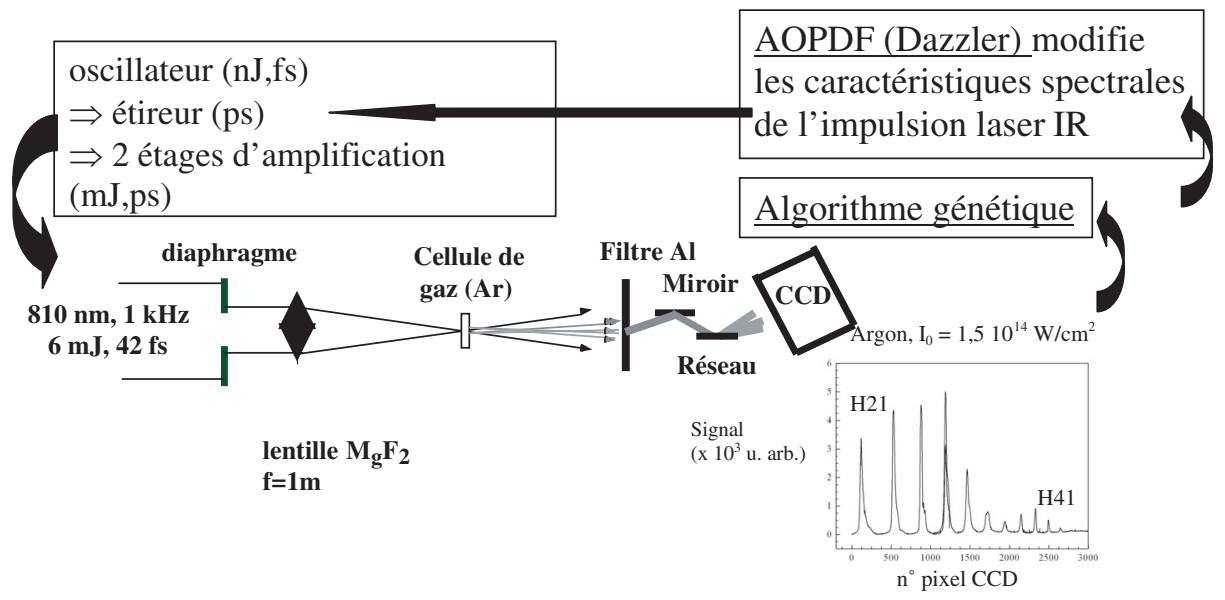

Figure 3. Dispositif expérimental.

pression... Pour l'harmonique 25 générée dans la cellule remplie de 22 mbar d'argon, l'optimum est obtenu pour un diaphragme de $18 \mathrm{~mm}$ de diamètre (énergie laser $=2 \mathrm{~mJ}$, longueur de Rayleigh $=$ $8,8 \mathrm{~mm}$ ) et pour une position de la cellule placée à $2 \mathrm{~mm}$ après le foyer du laser fondamental (Intensité crête dans la cellule environ $10^{14} \mathrm{~W} / \mathrm{cm}^{2}$ ). Avant chaque optimisation, nous vérifions à l'aide du SPIDER placé avant la lentille de focalisation que la phase spectrale est quasi-plate et proche de zéro (cf figure 5b).

Nous montrons sur la figure 4a un exemple d'optimisation du signal de trois harmoniques (H23, H25 et H27) pour chaque itération du programme. Le signal est augmenté d'un facteur 3 après 10 itérations. Après chaque optimisation, nous mesurons le profil temporel de l'impulsion, ainsi que la phase spectrale.

Une étude en fonction du diamètre du diaphragme montre que plus le diaphragme est ouvert, plus le front avant de l'impulsion optimisée présente une pente douce et la phase spectrale présente un $\varphi_{2}$ plus important (cf figure 5).
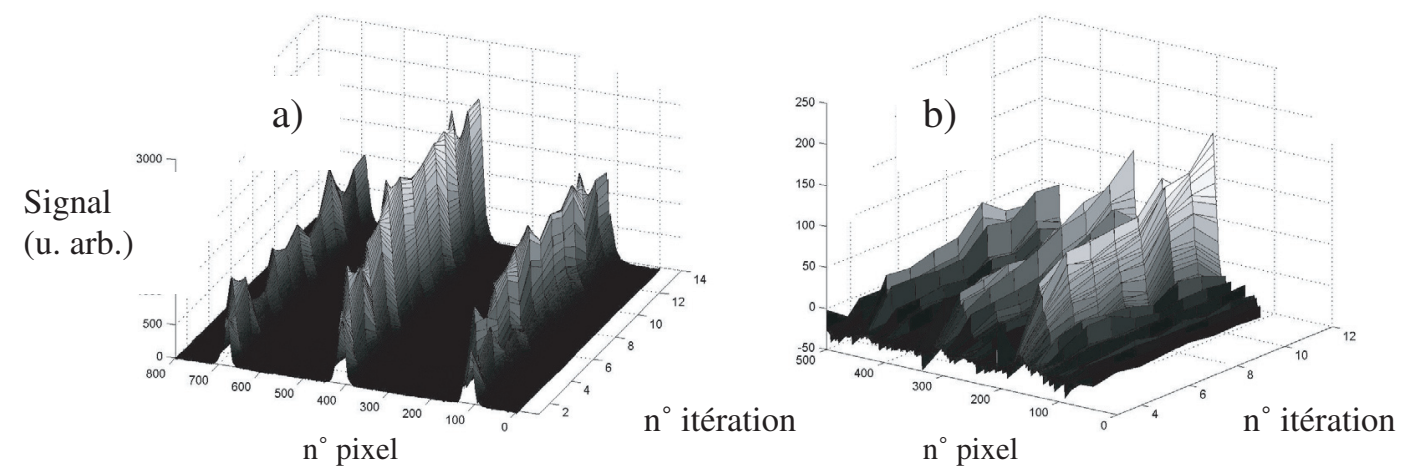

Figure 4. Spectres des harmoniques 23, 25 et 27(a) et des harmoniques 39, 41 et 43 (b) pour chaque itération de l'algorithme génétique.

L'algorithme génétique tend alors à diminuer l'intensité du laser dans le milieu générateur pour éviter une ionisation du milieu trop importante. En effet, l'énergie laser est augmentée d'un facteur 2 


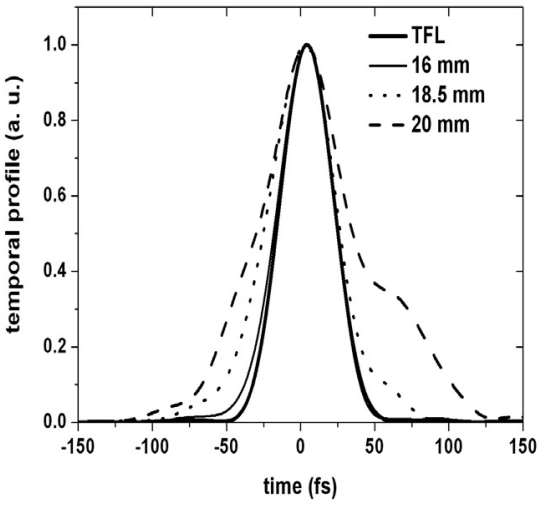

a)

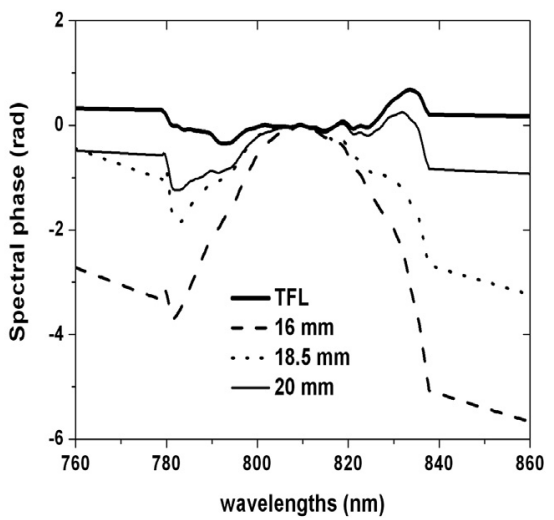

b)

Figure 5. Profil temporel (a) et phase spectrale (b) de l'impulsion laser IR après optimisation pour différents diamètres du diaphragme. Les courbes en trait plein épais correspondent à l'impulsion proche de la limite par Transformée de Fourier (phase spectrale quasi-plate).

lorsque le diamètre du diaphragme est augmenté de 16 à $25 \mathrm{~mm}$. Dans le même temps, la durée de l'impulsion est augmentée d'un facteur $2 / 3$ et passe de $42 \mathrm{fs}$ à $70 \mathrm{fs}$ entre 16 et $20 \mathrm{~mm}$. Pour un diamètre trop important, l'algorithme ne peut plus converger et le signal pour les harmoniques 25 et 27 est même plus faible après l'optimisation.

Pour les harmoniques plus élevées, nous avons mesuré une phase spectrale quasi-plate et proche de zéro après l'optimisation, ce qui correspond à une intensité dans la cellule plus élevée que pour les harmoniques plus basses. De plus, le facteur d'optimisation est plus important et peut même atteindre 10 pour l'harmonique 41 (cf figure $4 b$ ).

\section{3 Étude systématique}

Pour cette étude, nous avons fait varier la pression dans la cellule et la position de l'entrée de la cellule par rapport au foyer du laser IR. Ces deux paramètres jouent un rôle important pour l'accord de phase entre le faisceau d'une harmonique et le faisceau laser fondamental.

Nous remarquons sur la figure 6a que nous n'observons pas de maximum et nous n'avons pas atteint le régime de saturation dû à l'absorption des harmoniques dans le milieu. Pourtant, la longueur d'absorption pour H25 est inférieure à la longueur de la cellule dès 25 torr $\left(\mathrm{L}_{\text {cell }}=2 \mathrm{~mm}\right)$. Nous voyons aussi que lorsque la pression augmente, le rapport entre le signal après et avant l'optimisation est quasi constant et égal à 2 . Nous en déduisons que les dispersions électronique et atomique, importantes pour l'accord de phase, ne jouent pas de rôle dans ce processus d'optimisation.

Le résultat des optimisations en fonction de la position de l'entrée de la cellule par rapport au foyer du laser IR est présenté sur la figure 6b. Pour ces expériences, il faut noter que la distance de Rayleigh pour un diaphragme de $18 \mathrm{~mm}$ de diamètre est $8,8 \mathrm{~mm}$ (faisceau gaussien tronqué). Avant optimisation, nous observons un maximum lorsque le laser est focalisé à l'entrée de la cellule. Puis lorsque la cellule est placée à $8 \mathrm{~mm}$ du foyer, l'intensité est divisée de presque un facteur $2:$ l'harmonique 25 est alors dans la coupure et le signal chute. Après optimisation, nous montrons que le signal est optimum lorsque la cellule est placée $6 \mathrm{~mm}$ après le foyer. De plus, le rapport du signal après et avant optimisation augmente lorsque la cellule est placée loin du foyer, là où l'intensité est plus faible et où le gradient longitudinal d'intensité est important. Nous en déduisons que le processus d'optimisation dépend de ce gradient, via l'accord de phase. 


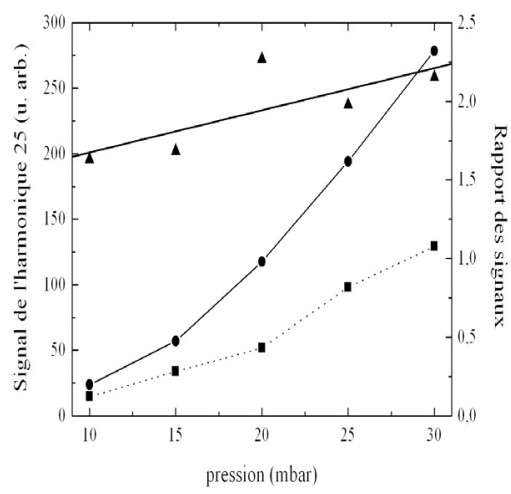

a)

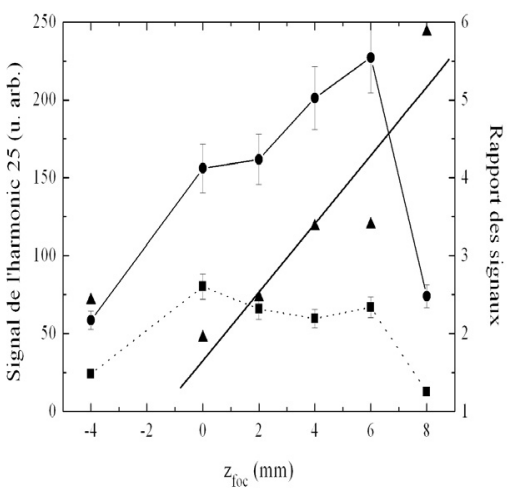

b)

Figure 6. Signal de la $25^{\text {è }}$ harmonique a) en fonction de la pression, b) en fonction de la position de l'entrée de la cellule de génération par rapport au foyer, avant (pointillés et carrés) et après l'optimisation (trait plein et rond). Le rapport des signaux est représenté avec des triangles et un ajustement en trait épais.

\section{DISCUSSION}

Nous rappelons que le signal harmonique dépend de la valeur du dipôle calculée pour un atome unique (réponse microscopique) et de l'accord de phase entre le faisceau fondamental et le faisceau harmonique (réponse macroscopique). Nous montrons tout d'abord l'optimisation qui dépend principalement de la réponse du dipôle à la forme temporelle de l'impulsion laser et les effets d'intensité. Puis nous discutons des effets d'accord de phase sur le processus d'optimisation.

La forme temporelle de l'impulsion joue principalement sur la réponse de l'atome unique car elle intervient dans le processus d'ionisation : lorsque le front avant est redressé, l'ionisation tunnel se produit pour des intensités plus importantes et le régime de saturation est atteint pour une intensité plus grande. Pour les harmoniques les plus élevées pour lesquelles l'intensité de coupure est élevée, le profil temporel de l'impulsion se rapproche de celui calculé à la limite de Fourier, c'est-à-dire pour une phase spectrale nulle. Par contre, pour les harmoniques autour de H25, il faut plutôt minimiser l'intensité pour ne pas trop ioniser le milieu. Ainsi l'algorithme génétique fait introduire via le Dazzler une dérive de fréquence linéaire importante $\left(\varphi_{2}\right)$ qui a pour but d'élargir l'impulsion. Pour un diaphragme trop ouvert ( $\phi=25 \mathrm{~mm}$ ), l'algorithme ne peut plus converger car l'augmentation de la dérive de fréquence est limitée par la taille du cristal du Dazzler $(90 \mathrm{~mm})$.

L' étude systématique en fonction de la pression du gaz et de la position de l'entrée de la cellule par rapport à la lentille a permis de montrer que les effets d'accord de phase sur le processus d'optimisation ne sont pas négligeables. Nous allons alors analyser ces effets.

Nous savons déjà que le facteur d'accord de phase introduit par S. Kazamias et al. [6] dépend du temps et est maximum à différents instants dans le front avant de l'impulsion laser suivant les conditions de génération. En particulier, plus l'intensité dans le milieu est faible, c'est-à-dire lorsque la cellule est placée loin du foyer, ce facteur est optimum pour une valeur du dipôle importante (cf figure 7). Par contre, lorsque la cellule est placée près du foyer, ce facteur est maximum dans l'aile avant de l'impulsion. Avec le Dazzler, nous avons alors la possibilité de modifier la forme temporelle du front avant et donc de contrôler l'instant d'optimisation de ce facteur par rapport au maximum de l'impulsion laser.

Une interprétation plus approfondie de ces résultats devra être réalisée afin de bien comprendre les mécanismes d'optimisation au niveau de la forme temporelle de l'émission harmonique. Ces expériences montrent la possibilité de contrôler le profil temporel du train d'impulsions attosecondes en agissant sur le profil temporel de l'impulsion IR. 


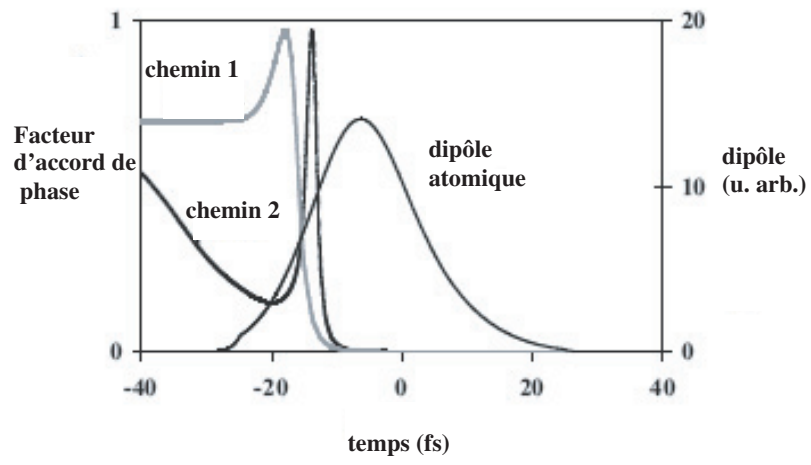

Figure 7. Facteur d'accord de phase en fonction du temps pour les chemins court (1) et long (2). La forme temporelle du dipôle atomique est aussi représentée (figure extraite de [6]).

\section{CONCLUSION ET PERSPECTIVES}

Nous avons montré dans cette étude expérimentale qu'il était possible d'optimiser le signal harmonique jusqu'à un facteur 10. Néanmoins, ce facteur est plus important pour les harmoniques très élevées, pour lesquelles la longueur d'absorption dépasse la longueur de la cellule. Cette étude expérimentale permet de dégager le comportement de la génération d'harmoniques en fonction du processus d'optimisation. En particulier, nous avons démontré que les couplages entre effets microscopique et macroscopique jouent un rôle important dans le processus d'optimisation.

D'autres voies sont explorées ou vont être explorées pour augmenter encore le signal harmonique et pour obtenir une source intense pour des longueurs d'onde plus faibles.

Nous avons aussi optimisé le signal harmonique en utilisant un miroir déformable à l'aide du même algorithme génétique. Nous avons atteint une optimisation d'un facteur 3 et nous interprétons ces résultats en termes de meilleur accord de phase entre le faisceau harmonique et le faisceau infrarouge. Ensuite, nous planifions d'utiliser une source laser $\mathrm{mJ}$ dont l'impulsion a été comprimée jusqu'à une durée d'impulsion inférieure à $10 \mathrm{fs}$ après filamentation dans une cellule d'argon [7]. Nous espérons atteindre des ordres plus élevés dans l'argon avec une efficacité de conversion aussi importante que pour les ordres plus bas [6].

\section{Remerciements}

Les auteurs tiennent à remercier Olivier Albert (LOA) et David Reitze (Univerrsité de Floride, USA) pour l'algorithme génétique et Denis Douillet (LOA) pour le montage expérimental et le spectromètre XUV.

\section{Références}

[1] R. Bartels et al., Nature 406, 164 (2000)

[2] Randy A. Bartels et al., Phys. Rev. A 70, 043404 (2004)

[3] David H. Reitze et al., Optics Lett. 29, 86 (2004)

[4] I. Iaconis and I. Walmsley, Opt. Lett. 23, 792 (1998)

[5] F. Verluise, V. Laude, Z. Cheng, Ch. Spielmann, and P. Tournois, Opt. Lett. 25, 575 (2000)

[6] S. Kazamias et al., Eur. Phys. J. D 26, 47 (2003)

[7] Ch. Hauri et al., Appl. Phys. B 79, 673 (2004) 